\title{
Barcodes galore for developmental biology
}

The emerging field of regenerative medicine has much to gain from basic research into the mechanisms of development, tissue regeneration and wound healing, all of which depend on the faithful execution of differentiation programs. Although these processes have been intensively studied for decades, a new class of methods based on Cas9 genome editing provides a welcome boost. In a recent paper in Science (Kalhor, R. et al. Science 361, eaat9804; 2018), Kalhor et al. show how genomic 'scarring' with Cas9 can be adapted to trace cell lineages during mouse embryo development.

The use of Cas9 for lineage tracing was pioneered by Shendure and colleagues (McKenna, A. et al. Science 353, aaf7907; 2016) in zebrafish. Their work, as well as subsequent improved methods, relied on errorprone repair of the double-strand breaks induced by Cas9. The small insertions and deletions introduced during repair act as barcodes; if several sites are cut at different time points during animal development, the accumulated errors can be used to reconstruct lineage trees.

The approach had previously been applied only in lower vertebrates and in cell culture. Because mouse development is more complex and more prolonged than that of the previously studied organisms, Kalhor et al. designed a system that increases the diversity of barcodes over longer time periods. To do so, they used so-called homing guide RNAs (hgRNAs), which direct Cas9 to a genomic locus encoding the hgRNA (Kalhor, R., Mali, P. \& Church, G.M. Nat. Methods 14, 195-200; 2017). This system enables continuous barcode evolution, because errors introduced in the targeting sequence also change the expressed sgRNA, which retains the ability to target its genomic locus.

Using a library of hgRNA constructs, Kalhor et al. generated a chimeric founder mouse, called mouse for actively recording cells 1 (MARC1), that carries 60 different hgRNA constructs in its genome. "Moving up the evolutionary ladder is a very important step and will allow addressing even more interesting questions," says Jan-Philipp Junker of the Max Delbrück Center in Berlin, Germany, "and the use of hgRNAs is a creative way to obtain the necessary barcode complexity."

To study early development, the authors crossed MARC1 with mice constitutively expressing Cas9. The different hgRNA sites were modified at different rates. 'Fast'
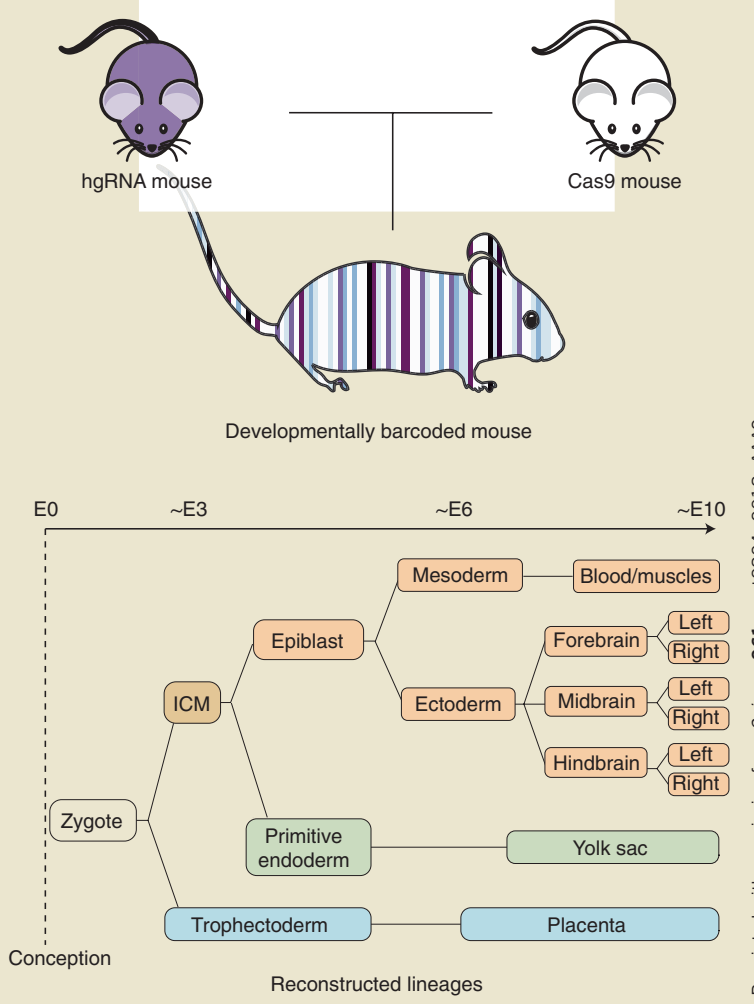

hgRNA sites were modified in almost all cells by E8.5, whereas 'slow' hgRNA sites were edited in only a minority of cells, even in adult animals. A group of intermediate hgRNAs that accumulated mutations throughout embryonic development were also observed. To validate the accuracy of the method, the authors reconstructed the first lineages in mouse development at embryonic day (E) 12.5. By sampling two tissues derived from each of the three basic developmental lineages, they accurately traced the differentiation of blastomeres into the embryonic inner cell mass and trophectoderm, as well as the subsequent differentiation of the inner cell mass into the primitive endoderm and epiblast, and of the trophectoderm into the placenta. Notably, a correct lineage tree could be constructed with as few as three hgRNAs in 50\% of cases. If only fast and intermediate hgRNAs were considered, three hgRNAs were sufficient to construct a correct tree in $90 \%$ of cases.

Kalhor et al. applied the method to address a longstanding question in mouse development. Both the anterior-posterior and the lateral axes are established in the embryonic neural tube by E8.5, although the order in which the axes emerge had been unclear. Dissecting the left and right sides of the cortex, cerebellum and tectum, the authors found that the barcodes of cells from the left and right side of each brain region were more similar to each other than to cells from any other brain regions, suggesting that the anterior-posterior axis is established before the lateral axes.

In principle, the diversity of repair outcomes (theoretically, $>10^{74}$ combinations of barcodes are possible for the 41 active hgRNAs in MARC1) is sufficient to uniquely label every single cell in an adult mouse as well as all the nodes of the developmental tree. Although the extent to which this diversity can be observed in practice remains to be seen, many open questions can clearly be addressed with this technique. "The logical next steps for the technology are to move to single-cell analysis to obtain lineage information of individual cell types and to couple it with inducible or tissue-specific Cas9 mice to investigate processes later in development or after experimental interventions," says Junker. Beyond development, applications in tumor development and metastasis may also be possible. Eventually, cell-type-specific barcodes might even reduce determination of the mouse brain connectome to a sequencing problem.

Markus Elsner, Senior Editor 